\title{
Effect of Right Ventricular Lead Placement on Tricuspid Valve: Added Value of Post-procedural Fluoroscopy to Three Dimensional Echocardiography in a Prospective Cohort Study
}

\author{
Hoorak Poorzand ${ }^{1}$, Mohammad Tayyebi ${ }^{2}$, Sara Hosseini ${ }^{1}$, Alireza Heidari ${ }^{1}$, Faeze \\ Keihanian $^{1}$, Lida Jarahi ${ }^{1}$, and Ali Hamedanchi ${ }^{1}$ \\ ${ }^{1}$ Mashhad University of Medical Sciences \\ ${ }^{2}$ Mashhad University of Medical Sciences Ghaem Hospital
}

August 17, 2020

\begin{abstract}
Introduction. Use of implantable endocardial electronic devices is widely increasing due to pro-longed life span of the community. Several studies evaluated the effect of right ventricular (RV) leads on tricuspid valve by three-dimensional transthoracic echocardiography (3D-TTE); howev-er, this affect has not yet been assessed by post-procedural fluoroscopy. Hence, the purpose of the current study was to evaluate the effect of RV lead placement on tricuspid valve, utilizing fluoroscopy in combination with 3D-TEE. Methods. We prospectively enrolled 59 patients who underwent clinically indicated pacemaker or implantable cardioverter defibrillator (ICD) implantation. Vena contracta (VC) and tricuspid regurgitation (TR) severity were measured using two-dimensional transthoracic echocardiography (2D-TTE) at baseline. Follow up 3D-TTE was performed six months after device implantation to assess TR severity and RV lead location. Results. TR VC was increased after the lead placement, compared to the baseline study (VC: $3.86 \pm 2.32$ vs $3.18 \pm 2.39 ; \mathrm{p}=0.005$ ). The mean changes in $\mathrm{VC}$ levels were $1.14 \pm$ $0.67 \mathrm{~mm}$ (Range: -0.4-2.5 mm) after inserting the lead. Among all investigated parameters, VC changes were predicted based on lead placement position only in 3D-TTE $(\mathrm{p}<0.001)$ while the other var-iables including fluoroscopy parameters were not predictive. Conclusion. The RV Lead location examined by 3D-TTE seems to be a valuable parameter to predict the changes in the severity of the tricuspid regurgitation. Fluoroscopy findings did not improve the predictive performance, at least in short term follow up.
\end{abstract}

\section{Hosted file}

final manuscript2.doc available at https://authorea.com/users/351503/articles/476097-effectof-right-ventricular-lead-placement-on-tricuspid-valve-added-value-of-post-proceduralfluoroscopy-to-three-dimensional-echocardiography-in-a-prospective-cohort-study 